\title{
Alimentação escolar nas comunidades quilombolas: desafios e potencialidades
}

\author{
School nutrition in 'quilombola' communities: \\ challenges and opportunities
}

Lucilene Maria de Sousa ${ }^{1}$ Karine Anusca Martins ${ }^{1}$ Mariana de Morais Cordeiro ${ }^{1}$ Estelamaris Tronco Monego ${ }^{1}$ Simoni Urbano da Silva ${ }^{1}$ Veruska Prado Alexandre ${ }^{1}$

${ }^{1}$ Faculdade de Nutrição, Universidade Federal de Goiás. Rua $227 \mathrm{~s} / \mathrm{n}^{\circ}$, Quadra 68, Setor Leste Universitário. 74605-080 Goiânia GO. lucilenemaria.sousa@ gmail.com

\begin{abstract}
The Brazilian School Nutrition Program (PNAE) is a Food and Nutritional Security (SAN) strategy for public school students. This article seeks to discuss the challenges and opportunities of school nutrition in 'quilombola' communities and report on the experience of the Cooperation Center for Student Food and Nutrition of the Federal University of Goiás and the Midwest Region (CECANE UFG/ Centro-Oeste). It includes a report on the experience with the systematization on PNAE, SAN and other policies. Continued access and adequate social policies are a challenge for the 'quilombola'* communities. Some economic, structural and social barriers have been identified in PNAE. In this context, Law 11.947/2009 encourages local development, with the acquisition of food from the region, and it establishes differentiated values per capita, which are translated into menus including products inherent to Afro-Brazilian culture that provide at least $30 \%$ of daily nutritional requirements. In the nutrition, health and quality of life project of 'quilombola'^ schoolchildren, the CECANE UFG/ Midwest carry out food and nutritional security actions. The area of school nutrition has proven responsive to local needs and supports the development and promotion of quality of life.
\end{abstract}

Key words School nutrition, Group with ancestors from the African continent, Public policies
Resumo O Programa Nacional de Alimentação Escolar (PNAE) é uma estratégia de Segurança Alimentar e Nutricional (SAN) para estudantes de escolas públicas. Este artigo tem por objetivos discutir os desafios e as potencialidades da alimentação escolar nas comunidades quilombolas e relatar a experiência do Centro Colaborador em Alimentação e Nutrição do Escolar da Universidade Federal de Goiás e Região Centro-Oeste (CECANE UFG/Centro-Oeste). Relato de experiência associado à sistematização da literatura sobre o PNAE, SAN e outras politicas. O acesso permanente e adequado às políticas sociais é um desafio para as comunidades quilombolas. Identificamse na execução do PNAE entraves de ordem econômica, estrutural e social. Neste contexto, a Lei 11.947/2009 incentiva o desenvolvimento local, por meio da aquisição de alimentos da região $e$ determina valor per capita diferenciado, traduzido em cardápio com alimentos da cultura negra e que atenda no mínimo 30\% das necessidades nutricionais diárias. O CECANE UFG/Centro-Oeste no projeto alimentação, saúde e qualidade de vida de escolares quilombolas realiza ações em SAN. O campo da alimentação escolar mostra-se sensivel às necessidades locais e apoia o desenvolvimento e a promoção da qualidade de vida.

Palavras-chave Alimentação escolar, Grupo com ancestrais do continente africano, Politicas públicas 


\section{Introdução}

No Brasil, o Programa Nacional de Alimentação Escolar (PNAE) é uma estratégia para a garantia do Direito Humano à Alimentação Adequada (DHAA), cujos pressupostos reportam aos princípios da alimentação saudável discutidos no Guia Alimentar para a População Brasileira ${ }^{1}$. Suas Diretrizes sugerem o respeito aos hábitos alimentares culturalmente referenciados, bem como o desenvolvimento local sustentável, traduzidas em duas ações: a educação alimentar e nutricional e a oferta de refeições que atendam às necessidades nutricionais de estudantes de escolas públicas durante o período letivo ${ }^{2,3}$.

A legislação vigente determina atendimento diferenciado aos escolares quilombolas como estratégia de promoção do DHAA. Trata-se de mecanismos de orçamento (com valor per capita diferenciado), planejamento (orientação para que o cardápio atenda às necessidades nutricionais específicas destes grupos e contribua para a superação da Insegurança Alimentar e Nutricional InSAN) e de garantia de geração de renda (prioridade na aquisição de alimentos oriundos da agricultura familiar destas comunidades, pela gestão do PNAE nos estados e municípios) ${ }^{2,3}$.

Comunidades quilombolas constituem grupos de indivíduos, cuja descendência tem relação com grupos étnico-raciais, segundo critérios de autoatribuição, com trajetória histórica própria, dotados de relações territoriais específicas, com presunção de ancestralidade negra relacionada com a resistência à opressão histórica sofrida. Para fins de acesso a direitos diversos, estas comunidades devem estar cadastradas na Fundação Cultural Palmares ${ }^{4}$.

No Brasil existem 1.711 comunidades quilombolas certificadas pela Fundação Cultural Palmares, das quais 7,7\% $(\mathrm{n}=131)$ na Região Sul; 9,2\% ( $\mathrm{n}=158)$ na Região Norte; $14,1 \%(\mathrm{n}=$ 241) na Região Sudeste; $62,8 \%(n=1075)$ na Região Nordeste e 6,2\% $(n=106)$ na Região Centro-Oeste ${ }^{5}$.

Embora reconhecidas e consideradas patrimônio cultural brasileiro, as comunidades quilombolas enfrentam graves problemas relacionados não só aos aspectos culturais, como à qualidade de vida e saúde de sua população. Estudos recentes em comunidades tradicionais e étnicas evidenciam a InSAN como um dos problemas de maior evidência nestas comunidades ${ }^{6-}$ ${ }^{9}$. Por esta razão, nos últimos anos diversas políticas públicas buscam garantir a interlocução das necessidades deste grupo populacional com as agendas governamentais, onde se destacam: o Plano Brasil sem Miséria ${ }^{10}$; a Política Nacional de Saúde Integral da População Negra ${ }^{11}$ e o Programa Brasil Quilombola ${ }^{12}$. O PNAE está inserido no escopo destas políticas, sendo as ações propostas reiteradas pelo Plano Nacional de Segurança Alimentar e Nutricional ${ }^{13}$.

Nesta perspectiva, este estudo tem como objetivo discutir os desafios e as potencialidades da alimentação escolar nas comunidades quilombolas e relatar a experiência do Centro Colaborador em Alimentação e Nutrição do Escolar da Universidade Federal de Goiás e Região CentroOeste (CECANE UFG/Centro-Oeste) junto ao PNAE quilombola no estado de Goiás. Este estudo foi aprovado pelo Comitê de Ética em Pesquisa da Universidade Federal de Goiás.

\section{O contexto das comunidades quilombolas}

Embora a preocupação com a qualidade de vida esteja refletida nas políticas públicas no Brasil, inquérito nacional realizado pelo Ministério do Desenvolvimento Social e Combate à Fome (MDS) em comunidades quilombolas indica que a maioria destas famílias encontra-se em situação de extrema pobreza. Apenas 29\% têm acesso ao serviço de coleta de lixo; $24,0 \%$ ao esgotamento sanitário e $56,0 \%$ à água encanada ${ }^{9}$. De forma similar, estudos realizados em comunidades do Tocantins, Pará e Paraíba evidenciam que a InSAN esteve presente em $85,0 \%$ das famílias ${ }^{6}$ com uma frequência elevada de domicílios de adobe, palhoça ou taipa ${ }^{7,8}$.

Em relação às iniciativas econômicas e produtivas, a produção de alimentos por meio da agricultura tem destaque nestas comunidades, sendo prática usual para $93 \%$ das famílias, seguida pela pecuária $(56,0 \%)$ e pela pesca $(32,0 \%)^{14}$. A maior parte dos produtos provenientes destas atividades é utilizada como subsistência, com uma comercialização reduzida, seja por dificuldades de acesso ou no transporte ${ }^{8,9}$.

A maioria das comunidades $(89,0 \%)$ dispõe de escolas ${ }^{9}$, porém, estudo realizado na Paraíba evidenciou que apenas 34,5\% das crianças quilombolas estudam 7 . Em adição a esta informação, 43,6\% das mães das crianças quilombolas de zero a cinco anos têm até quatro anos de escolaridade, enquanto $47,0 \%$ dos chefes de família estudou até o quarto ano do ensino fundamental, evidenciando altos índices de analfabetismo funcional ${ }^{9}$.

Estudo denominado Chamada Nutricional Quilombola ${ }^{9}$, investigou o estado nutricional de 
2.723 crianças menores de cinco anos, das quais $11,6 \%$ e $8,1 \%$ apresentaram déficit de Altura/Idade e de Peso/Idade, respectivamente, sendo que as famílias menos favorecidas socioeconomicamente apresentaram uma pior situação nutricional.

Em relação ao consumo alimentar, foi observado que a maioria das crianças de três a 11 anos e 7,5\% da população de 11 anos e mais consumiam menos do que três refeições por dia, indicativo da presença de InSAN em famílias quilombolas 9 . De forma similar, estudo realizado em 14 comunidades quilombolas do Tocantins observou reduzido consumo de alimentos tanto entre adultos quanto em crianças, com cerca de 15,0\% de InSAN grave neste grupo social ${ }^{6}$.

Diante deste cenário, é possível supor que o PNAE seja uma estratégia de enfrentamento da InSAN entre os estudantes quilombolas. Porém, estudo realizado em comunidade do Pará evidenciou irregularidades na entrega, além de alimentos inadequados ao hábito dos estudantes, sendo comum a oferta de alimentos semiprontos como sopas e mingaus ${ }^{8}$. Diagnóstico preliminar realizado pela equipe do CECANE UFG/ Centro-Oeste em visita realizada a comunidade quilombola de Goiás, indicou a presença de produtos industrializados alheios aos hábitos regionais, contrapondo-se ao grande potencial agrícola para produção de frutas e hortaliças, que não estão presentes na alimentação escolar ${ }^{15}$.

\section{Políticas públicas, o Direito Humano à Alimentação Adequada e as comunidades quilombolas}

A Política Nacional de Segurança Alimentar e Nutricional (PNSAN) ${ }^{16}$ a Lei Orgânica de Segurança Alimentar e Nutricional (LOSAN) ${ }^{17}$, a Política Nacional de Alimentação e Nutrição $(\mathrm{PNAN})^{18}$ e a legislação do PNAE ${ }^{2,3}$ estabelecem Diretrizes e articulam diferentes setores da sociedade civil e órgãos governamentais em ações universais e locais em prol da efetivação do DHAA no território brasileiro.

Do ponto de vista operacional, a PNSAN traduz-se no Plano Nacional de Segurança Alimentar e Nutricional, cuja finalidade é a concretização do DHAA e a construção de um modelo sustentável de produção e consumo de alimentos no país, preconizado pelo Sistema Nacional de Segurança Alimentar e Nutricional (SISAN) ${ }^{16}$. Dentre as Diretrizes do Plano evidenciam-se avanços relativos à garantia de direitos dos povos e comunidades tradicionais, com destaque para a promoção, a universalização e a coorde- nação das ações de Segurança Alimentar e Nutricional $(\mathrm{SAN})^{13}$.

Na prática, o planejamento e a execução das ações coordenadas de SAN desdobram-se em articulações locais, tendo como referencial os territórios destas comunidades tradicionais, onde o Setor Educação, incluídas as unidades escolares, é chamado a garantir a oferta regular e permanente de alimentos com atenção à identidade cultural dos estudantes quilombolas. Entretanto, a concretização desta responsabilidade se dá por meio de articulações com outros setores, como o agrícola (buscando assistência técnica referenciada nas tradições culturais de cultivo de alimentos e o resgate das formas tradicionais de cultivo, incluindo aqui o uso de sementes crioulas e animais da região) e o setor saúde (com monitoramento dos indicadores de InSAN), dentre outros.

Com o objetivo de reduzir os desafios na articulação entre os setores, as Diretrizes do PNSAN indicam ações capazes de promover a interface da alimentação escolar com outras necessidades das comunidades quilombolas. Por esta razão, sugerem o aprimoramento, a qualificação e o monitoramento da execução do PNAE nas escolas localizadas em comunidades quilombolas; a expansão da participação de agricultores(as) familiares quilombolas nos mercados institucionais; a garantia da regularização fundiária e a certificação das comunidades quilombolas; além da proteção e estímulo da agrobiodiversidade e do desenvolvimento local sustentável ${ }^{13}$.

O Plano estabelece metas concretas e monitoráveis para as ações e programas de SAN, e, nesta perspectiva, tem como um dos objetivos o fortalecimento da PNAN, cujo propósito é melhorar as condições de alimentação, nutrição e saúde, em busca da garantia da SAN da população brasileira. A PNAN, aprovada no ano de 1999 e reformulada entre 2010 e 2011, é pautada nos Princípios do Sistema Único de Saúde e do DHAA, com destaque ao respeito à diversidade e à cultura alimentar, o fortalecimento da autonomia dos indivíduos e a busca da segurança alimentar e nutricional com soberania ${ }^{18}$.

Tendo as comunidades quilombolas como eixo de discussão, identificam-se nas Diretrizes do Plano de SAN convergências com as reivindicações e as políticas voltadas a este grupo ${ }^{13}$ :

- Aprimoramento, qualificação e acompanhamento da alimentação escolar nas escolas situadas em comunidades quilombolas; fomento à aquisição, pelas escolas, de gêneros alimentícios da agricultura familiar quilombola; distribuição de alimentos às famílias vulneráveis à 
fome, dentre estas, aquelas situadas em comunidades remanescentes de quilombos [Diretriz 1];

- A ampliação do acesso aos serviços de assistência técnica e extensão rural para os agricultores familiares quilombolas, com conseqüente aumento da participação dos mesmos no abastecimento de mercados institucionais [Diretriz 2];

- Regularização fundiária/certificação das comunidades quilombolas e promoção da saúde e SAN dos povos quilombolas, por meio do uso sustentável da agrobiodiversidade [Diretriz 4];

- A ampliação da cobertura de ações e serviços de saneamento básico e de abastecimento de água em comunidades quilombolas, priorizando soluções alternativas que permitam a sustentabilidade dos serviços [Diretriz 6].

Ao evidenciar a priorização de ações do Estado, com foco na população quilombola, estas políticas mostram sintonia com os condicionantes de renda, concentração da propriedade de terra, dificuldade de acesso ao alimento, dentre outros, que historicamente determinaram uma alta prevalência de InSAN nestas comunidades.

\section{Alimentação escolar \\ nas comunidades quilombolas de Goiás: para além da oferta de alimento}

Pelo exposto, é possível supor que as últimas décadas representaram um avanço, seja no aumento da visibilidade das questões relacionadas à qualidade de vida das comunidades quilombolas, bem como nas políticas de apoio a este segmento. O desafio é compatibilizar o proposto ao executado, em especial pelas políticas relacionadas à comunidade escolar e ao $\mathrm{PNAE}^{2,3}$ nas comunidades quilombolas. A ineficiência na implementação local, em especial a irregularidade da oferta de alimentos saudáveis e adequados, distantes dos hábitos regionais; a inadequação na distribuição dos recursos financeiros destinados ao Programa; as dificuldades na logística de distribuição, armazenamento e produção dos alimentos oferecidos aos escolares quilombolas, são questões centrais nesta discussão ${ }^{19}$.

Neste contexto, a Lei 11.947/2009 representa um avanço por destacar em suas Diretrizes o apoio ao desenvolvimento sustentável, com incentivo para a aquisição de gêneros alimentícios diversificados, produzidos em âmbito local, com destaque no Artigo 14 que indica no mínimo $30,0 \%$ dos recursos financeiros repassados pelo Fundo Nacional de Desenvolvimento da Educação (FNDE), no âmbito do PNAE, deverá ser utilizado na aquisição de gêneros alimentícios provindos diretamente da agricultura familiar e do empreendedor familiar rural ou de suas organizações, priorizando-se os assentamentos da reforma agrária, as comunidades tradicionais indígenas e comunidades quilombolas ${ }^{2}$. A inclusão de agricultores quilombolas neste mercado institucional é fundamental para o desenvolvimento local sustentável e para a possível superação da InSAN em suas comunidades.

O benefício da alimentação para escolares quilombolas inclui recurso financeiro diferenciado para execução do PNAE. O valor per capita atualmente repassado aos municípios com estudantes quilombolas matriculados no ensino fundamental e médio, além de jovens e adultos (EJA), é de R $\$ 0,60$ (sessenta centavos de real), enquanto para os demais estudantes é de R $\$ 0,30$ (trinta centavos de real ${ }^{20}$. Já o cardápio elaborado para este grupo de estudantes deve atingir no mínimo 30,0\% das necessidades nutricionais diárias, superior em $10,0 \%$ ao estipulado para o cardápio destinado aos demais alunos da rede pública de educação matriculados em período parcial $^{3}$.

O CECANE-UFG/Centro-Oeste é um espaço de fortalecimento e consolidação de políticas públicas em alimentação e nutrição, com ênfase na SAN e no DHAA, cujo foco é o ambiente escolar. As comunidades quilombolas são parte deste universo, portanto, inseridas neste contexto. Um dos projetos em execução pelo CECANE-UFG/ Centro-Oeste denomina-se Alimentação, saúde e qualidade de vida de escolares quilombolas, cujas atividades iniciaram-se em 2010, com a identificação de lideranças e entidades articuladas com populações negras, povos e comunidades tradicionais. Este grupo elaborou coletivamente um projeto de ação, a partir de uma oficina que levantou os temas de maior relevância, categorizados em oito possibilidades de ação, a partir de então considerados como a espinha dorsal do projeto:

(1) Condições de saúde e nutrição

Investigação sobre como vivem, adoecem e morrem os indivíduos das comunidades quilombolas, com vistas a identificar a situação de saúde, nutrição e qualidade de vida.

\section{(2) Infraestrutura}

Avaliação das condições das estradas; da estrutura física das escolas (unidades de alimentação e nutrição) e domicílios; do saneamento básico (acesso a energia elétrica, água, esgoto e lixo) e o estudo da cadeia de transporte do alimento para a escola.

(3) Gestão

Estudo da aplicação do recurso do PNAE quilombola; identificação do tipo de cardápio da zona 
urbana e rural, além da avaliação da atuação do manipulador de alimentos e nutricionista.

\section{(4) Controle social}

Identificação do Conselho de Alimentação Escolar (CAE) e suas ações; mapeamento de outras organizações sociais na região, tais como cooperativas e associações.

\section{(5) Agricultura familiar}

Estudo da produção (tipo de alimento produzido e beneficiamento) e da comercialização (local e para o mercado externo), além do conhecimento do agricultor familiar da Lei 11.947/ 2009.

(6) Alimentos e alimentação escolar

Avaliação do cardápio e dos alimentos oferecidos (qualidade, quantidade e regularidade); estudo da cultura alimentar local e sua inserção no cardápio e currículo escolar.

(7) Educação permanente

Nos municípios estudados, identificação do processo de formação de manipuladores de alimentos, nutricionista, CAE, agricultor familiar e gestores, dentre outros.

\section{(8) Mundo do trabalho}

Identificação de alternativas de apoio aos jovens e adultos em atividades cujo objeto seja a manutenção dos mesmos junto a suas comunidades.

Promover a SAN junto às comunidades quilombolas é um grande desafio, sobretudo pelas peculiaridades destas comunidades. Neste contexto, a identificação de parceiros em nível local, a compreensão deste processo como sendo de caráter interssetorial, e a necessária aproximação e diálogo com as lideranças locais apresentam potencialidade para que se constitua um espaço coletivo de construção e aprendizagens mútuas.

O projeto está em execução, com o estabelecimento de metas que, no curto prazo (segundo semestre/2011), traçou ações emergenciais e de aproximação com as comunidades, com destaque para as oficinas de sensibilização para a questão do PNAE, voltada para agricultores e estudantes quilombolas e a participação na ação $\mathrm{Ka}$ lunga Cidadão, atividade articulada com diversas áreas do conhecimento da Universidade Federal de Goiás. No médio prazo (primeiro semestre/ 2012) ocorreu uma oficina para validação do instrumento, posteriormente aplicado nas 22 comunidades quilombolas goianas certificadas pela Fundação Cultural Palmares. No longo prazo (primeiro semestre/2013) estão previstas reuniões locais para divulgação dos resultados e pactuação de ações de intervenção.
A aproximação dos saberes popular e científico, permitindo uma inserção no cotidiano das comunidades quilombolas possibilita a construção de propostas de ações com potencial para modificar a realidade local, e tem permitido à equipe do CECANE UFG/Centro-Oeste vivenciar aprendizados, conquistas e muitos desafios.

Dentre os aprendizados, destacamos a construção compartilhada da proposta com membros das comunidades quilombolas de Goiás; representantes de organizações sociais e órgãos governamentais envolvidos na temática do projeto. E a relevância da aproximação com os sujeitos da ação, possibilitando assim a identificação de diferentes necessidades que condicionam a InSAN neste grupo social e como estes se expressam no cotidiano destas comunidades.

Os avanços contemplam a realização de oficinas nos territórios das comunidades quilombolas, para discutir com os agricultores familiares a comercialização dos gêneros alimentícios produzidos para a alimentação escolar, atendendo ao proposto na legislação do PNAE; bem como a discussão com os escolares sobre o consumo alimentar no ambiente escolar e familiar, ambas com boa repercussão entre os participantes.

Por outro lado, os desafios existem e são tão complexos quanto a realidade deste grupo social, seja na tradução do conhecimento produzido em uma linguagem compreensível aos sujeitos da ação; seja no desafio de compreender que um silêncio, um olhar, uma palavra, são sinais de que há desejo de mudanças.

Quando a gente tem o conhecimento, a gente tem como cobrar expressou uma representante de comunidade quilombola presente em um de nossos encontros ${ }^{15}$. Este é o nosso maior desafio: enquanto grupo que detém o saber técnico, colocá-lo a serviço da promoção e proteção do DHAA e SAN nas comunidades quilombolas, num cenário de dificuldades no acesso a bens e políticas sociais.

\section{Colaboradores}

LM Sousa, KA Martins, MM Cordeiro, ET Monego, SU Silva e VP Alexandre participaram de todas as etapas de elaboração do manuscrito: concepção, desenho, proposição de ideias, análise crítica e redação do manuscrito. 


\section{Referências}

1. Brasil. Ministério da Saúde (MS). Guia Alimentar para a população brasileira: promovendo a alimentação saudável [Internet]. Brasília: MS; 2005. [acessado $2011 \mathrm{dez}$ 12]. Disponível em: http://dtr2001. saude.gov.br//produtos/livros/pdf/05_1109_M.pdf.

2. Brasil. Lei $n^{\circ} 11.947$, de 16 de junho de 2009. Dispõe sobre o atendimento da alimentação escolar e do Programa Dinheiro Direto na Escola aos alunos da educação básica; altera as Leis nos 10.880, de 9 de junho de 2004, 11.273, de 6 de fevereiro de 2006, 11.507, de 20 de julho de 2007; revoga dispositivos da Medida Provisória no 2.178-36, de 24 de agosto de 2001, e a Lei no 8.913, de 12 de julho de 1994; e dá outras providências. Diário Oficial da União 2009; 17 jun.

3. Brasil. Fundo Nacional de Desenvolvimento da Educação. Resolução/FNDE/CD/No 38, de 16 de julho de 2009. Dispõe sobre o atendimento da alimentação escolar e do Programa Dinheiro Direto na Escola aos alunos de educação básica e dá outras providências. Diário Oficial da União 2009; 17 jul.

4. Brasil. Presidência da República. Decreto n ${ }^{\circ} 4.887 /$ 2003, de 20 de novembro de 2003. Regulamenta o procedimento para identificação, reconhecimento, delimitação, demarcação e titulação das terras ocupadas por remanescentes das comunidades dos quilombos de que trata o art. 68 do Ato das Disposições Constitucionais Transitórias. Brasília; 2003. Diário Oficial da União 2003; 21 nov.

5. Brasil. Ministério da Cultura. Fundação Cultural Palmares [Internet]. Comunidades Quilombolas. [acesso $2011 \mathrm{dez} 2011$ ]. Disponível em: http:// www.palmares.gov.br/?page_id=88.

6. Monego ET, Peixoto MRG, Cordeiro MM, Costa RM. (In)segurança alimentar em comunidades quilombolas do Tocantins. Segurança Alimentar e Nutricional. 2010; 17(1):37-47.

7. Silva JANS. Condições sanitárias e de saúde em Caiana dos Crioulos, uma comunidade quilombola do estado da Paraíba. Saúde Soc. 2007; 16(2):111-124

8. Oliveira e Silva D, Guerrero AFH. Guerrero $\mathrm{CH}$, Toledo LMA. A rede de causalidade da insegurança alimentar e nutricional de comunidades quilombolas com a construção da rodovia BR-163, Pará, Brasil. Rev. Nutr. 2008; 21(Supl.):83-97.

9. Brasil. Ministério do Desenvolvimento Social e Combate à Fome (MDS). Políticas Sociais e Chamada Nutricional Quilombola: estudos sobre condições de vida nas comunidades e situação nutricional das crianças. Cadernos de Estudos Desenvolvimento Social em Debate, no 9. Brasília: MDS; 2008.

10. Brasil. Presidência da República. Decreto $n^{\circ} .7 .492$ de 02 de junho de 2011. Institui o Plano Brasil Sem Miséria. Diário Oficial da União 2011; 3 jun.

11. Brasil. Ministério da Saúde (MS). Política Nacional de Saúde Integral da População Negra [Internet]. Brasília: MS; 2007. [acessado 2011 nov 28]. Disponível em: http://bvsms.saude.gov.br/bvs/publicacoes/ politica_nacional_saude_populacao_negra.pdf.

12. Brasil. Secretaria Especial de Políticas de Promoção da Igualdade Racial. Programa Brasil Quilombola. Brasília: Abaré; 2005. [acessado 2011 nov 28]. Disponível em: http://bvsms.saude.gov.br/bvs/publicacoes/ brasilquilombola_2004.pdf
13. Brasil. Câmara Interministerial de Segurança Alimen tar e Nutricional. Plano Nacional de Segurança Alimentar e Nutricional: 2012/2015. Brasília: Ministério do Desenvolvimento Social e Combate à Fome; 2011. [acessado $2011 \mathrm{dez} 10$ ]. Disponível em: http://www. mds.gov.br/gestaodainformacao/biblioteca/secretarianacional-de-seguranca-alimentar-e-nutricional-sesan/ livros/plano-nacional-de-seguranca-alimentar-enutricional-2012-2015/plano-nacional-de-segurancaalimentar-e-nutricional-2012-2015.

14. Pedroso Júnior NN, Murrieta RSS, Taqueda CS, Navazinas ND, Ruivo AP, Bernardo DV, Neves WA. A casa e a roça: socieconomia, demografia e agricultura em populações quilombolas do Vale do Ribeira, São Paulo, Brasil. Bol. Mus. Para. Emilio Goeldi Cienc. Hum. 2008; 3(2):227-252.

15. Cordeiro MM, Monego ET, Alexandre VP. Relatório final: (In)segurança alimentar e nutricional em comunidades quilombolas e a execução do PNAE. Goiânia: Centro Colaborador em Alimentação e Nutrição do Escolar da Universidade Federal de Goiás e Região Centro Oeste; 2011.

16. Brasil. Presidência da República. Decreto $n^{\circ} 7272$, de 25 de agosto de 2010. Regulamenta a Lei $n^{\circ} 11.346$ de 15 de setembro de 2006 que cria o Sistema de Segurança Alimentar e Nutricional [SISAN] como vistas a assegurar o Direito Humano à Alimentação Adequada e institui a Política Nacional de Segurança Alimentar e Nutricional. Diário Oficial da União 2010; 26 ago.

17. Brasil. Presidência da República. Lei no 11.346 , de 15 de setembro de 2006. Cria o Sistema Nacional de Segurança Alimentar e Nutricional - SISAN com vistas em assegurar o direito humano à alimentação adequada e dá outras providências. Diário Oficial da União 2006; 18 set.

18. Brasil. Ministério da Saúde (MS). Política Nacional de Alimentação e Nutrição [Internet]. Brasília: MS 2011. [acessado $2011 \mathrm{dez} 13$ ]. Disponível em: http:// 189.28.128.100/nutricao/docs/geral/pnan2011.pdf. Leite IB. O projeto político quilombola: desafios, con-

19. quistas e impasses atuais. Revista Estudos Feministas [Internet]. 2008. [acessado $2011 \mathrm{dez} 11$ ]; 16(3):965 977. Disponível em: http://www.periodicos.ufsc.br/ index.php/ref/article/view/9951/9189.

Brasil. Fundo Nacional de Desenvolvimento da

20. Educação. Resolução/FNDE/CD/Nº 67, de 28 de dezembro de 2009. Altera o valor per capita para oferta da alimentação escolar do Programa Nacional de Alimentação Escolar - PNAE. Diário Oficial da União 2009; 29 dez.

Artigo apresentado em 03/01/2013

Aprovado em 05/01/2013

Versão final apresentada em 08/01/2013 